COLO-HEP-420

\title{
The correlation length of the Heisenberg antiferromagnet with arbitrary spin $\mathrm{S}$
}

\author{
Peter Hasenfratd \\ Department of Physics \\ University of Colorado at Boulder \\ Boulder, CO 80309-0390
}

October 2018

\begin{abstract}
Experiments and numerical data on the correlation length $\xi(T)$ for large $S$ disagree strongly with the theoretical prediction based on the effective field theory prescription of the magnon physics. The reason is that for large $S$, at any accessible $\xi(T)$, the cut-off effects from the nonmagnon scales become large and can not be treated by an effective field theory. We study these effects in a spin-wave expansion. The corrected prediction on $\xi(T)$ connects the renormalized classical and the classical scaling regions smoothly and comes close to the data.
\end{abstract}

\footnotetext{
${ }^{1}$ On leave from the Institute of Theoretical Physics, University of Bern, Sidlerstrasse 5, CH-3012 Bern, Switzerland.
} 
There exist a number of quasi-2D antiferromagnetic compounds, including spin- $1 / 2$ antiferromagnets (AFMs) $\mathrm{La}_{2} \mathrm{CuO}_{4}$ and $\mathrm{Sr}_{2} \mathrm{CuO}_{2} \mathrm{Cl}_{2}$ [1], spin- 1 AFMs $\mathrm{La}_{2} \mathrm{NiO}_{4}$ and $\mathrm{K}_{2} \mathrm{NiF}_{4}$ [2] and spin-5/2 AFM Rb $\mathrm{MnF}_{4}$ [3, 4, 5] whose magnetic behavior is well described by the $2 \mathrm{D}$ quantum Heisenberg model[

$$
H=J \sum_{n, i} \mathbf{S}_{n+\hat{\imath}} \mathbf{S}_{n}, \quad \mathbf{S}_{n}^{2}=S(S+1) .
$$

The low temperature properties of this model are dominated by magnon excitations and can be described by an $O(3)$ invariant effective field theory [6]-[9] whose leading part contains two parameters only: $\rho_{s}$ (spin stiffness) and $c$ (spinwave velocity)

$$
\mathcal{A}_{\text {eff }}^{\text {leading }}=\frac{\rho_{s}}{2 c} \int_{0}^{c / T} d \tau \int d^{2} x \partial_{\mu} \mathbf{R}(\tau, x) \partial_{\mu} \mathbf{R}(\tau, x), \quad \mathbf{R}^{2}=1,
$$

where $\mu=0,1,2$ refer to $\tau, x_{1}, x_{2}$, respectively. In their work[10], Chakravarty, Halperin and Nelson have used renormalization group to connect this effective theory with the $d=2$ classical $O(3)$ non-linear $\sigma$-model and, among other results, predicted the asymptotic, small temperature behavior of the correlation length $\xi \sim c / 2 \pi \rho_{s} \exp \left(2 \pi \rho_{s} / T\right)$. The exact mass gap of the $\sigma$-model[1] and the two-loop corrections lead finally to the asymptotic expression 12

$$
\xi=\frac{e}{8} \frac{c}{2 \pi \rho_{s}} \exp \left(\frac{2 \pi \rho_{s}}{T}\right)\left(1-\frac{T}{4 \pi \rho_{s}}+O\left(T^{2}\right)\right) .
$$

It has been demonstrated in a careful numerical study 13] that the explicitly given terms in eq. (3) are consistent with the numerical data at very large correlation lengths (low temperatures) for $S=1 / 2$. At moderate correlation lengths, however experiments[1]-[5] numerical data 13]- 116], series expansions [17 and a semiclassical model 18] indicate a significant discrepancy which is increasing rapidly with $S$. It was subsequently realized[19, 20, 17] that the quantum Heisenberg model contains a sequence of crossovers (depending on $T$ and the parameters $J$ and $S$ ), and eq. (3) is valid only in a corner called the region of 'renormalized classical scaling'.

This situation is unsatisfactory since the basic feature of the quantum Heisenberg model in eq. (1), namely that it can be mapped onto a simpler model, is valid beyond the region of renormalized classical scaling. The purpose of this paper is to determine the corrections to eq. (3) making it applicable at moderate correlation lengths also and for any value of $S$ including the large $S$ limit ('classical scaling region').

We calculated the corrections to eq. (3) (which are due to cut-off effects in the quantum Heisenberg model) in leading non-trivial order of spin-wave expansion

\footnotetext{
${ }^{2}$ We use $\hbar=1, k_{\mathrm{B}}=1$ convention.
} 


\begin{tabular}{||l|l|l|l|l|l||}
\hline$\gamma$ & $\exp (-C)$ & $\gamma$ & $\exp (-C)$ & $\gamma$ & $\exp (-C)$ \\
\hline 0.0125 & 2.1718 & 1.2 & 0.4280 & 4.5 & 0.9410 \\
0.025 & 1.1341 & 1.4 & 0.4934 & 5.0 & 0.9538 \\
0.05 & 0.6180 & 1.5 & 0.5256 & 5.5 & 0.9623 \\
0.1 & 0.3658 & 1.75 & 0.6021 & 6.0 & 0.9691 \\
0.2 & 0.2528 & 2.0 & 0.6704 & 7.0 & 0.97771 \\
0.4 & 0.2288 & 2.25 & 0.7288 & 8.0 & 0.98311 \\
0.5 & 0.2399 & 2.5 & 0.7775 & 10.0 & 0.98931 \\
0.6 & 0.2576 & 3.0 & 0.8484 & 15.0 & 0.99529 \\
0.8 & 0.3055 & 3.5 & 0.8936 & 20.0 & 0.99736 \\
1.0 & 0.3640 & 4.0 & 0.9223 & 30.0 & 0.99883 \\
\hline
\end{tabular}

Table 1: The correcting factor $\exp (-C)$ as the function $\gamma=2 J S / T$.

and obtained

$$
\xi^{\prime}=\frac{e}{8} \frac{c}{2 \pi \rho_{s}} \exp \left(\frac{2 \pi \rho_{s}}{T}\right)\left(1-\frac{T}{4 \pi \rho_{s}}\right) \exp (-C(\gamma)), \quad \gamma=\frac{2 J S}{T},
$$

where the correction factor $\exp (-C(\gamma))$ is given in Table 1 for different $\gamma$ values. Here $\gamma=2 J S / T \sim \Lambda^{\text {cut }} / T(1+O(1 / S))$, where $\Lambda^{\text {cut }}=c / a$ and $a$ is the lattice unit. In the limit $T \rightarrow 0$, ( $S$ fixed), we have $\gamma \rightarrow \infty$, and $C \sim \gamma^{-2}$, leading to the old result in eq. (3). For large $S$ and $\rho_{s} \gg T \gg \Lambda^{\text {cut }}$ (classical scaling region), we have $\gamma \rightarrow 0$ and $C(\gamma)=\pi / 2+\ln 8+\ln \gamma+O(\gamma)$ which gives

$$
\frac{1}{a} \xi_{\text {classical }}=\frac{\exp \left(-\frac{\pi}{2}\right)}{\sqrt{32}} \frac{e}{8} \frac{T}{2 \pi \rho_{s}} \exp \left(\frac{2 \pi \rho_{s}}{T}\right)\left(1-O\left(\frac{\Lambda^{\text {cut }}}{T}\right)\right),
$$

where $\rho_{s} \sim J S^{2} \sim \rho_{\text {class }}$. The fact that the prefactor $\exp (-\pi / 2) / \sqrt{32}$ comes out automatically from the corrections is a non-trivial test on our result. ${ }^{3}$ For intermediate $\gamma$ values, $\exp (-C(\gamma))$ gives a significant factor as shown in fig. 1, where, for correlation lengths larger than 10 , the corrected theoretical prediction is compared with $\mathrm{MC}$ and series expansion data and with a semiclassical model.'.

We shall first discuss the steps leading to eq. (4), then we close the paper with a few remarks.

\footnotetext{
${ }^{3} \mathrm{Eq}$. (5) has the expected form 20 : for $S \rightarrow \infty$, the quantum Heisenberg model goes over to the standard lattice regularized $2 \mathrm{D} O(3) \sigma$-model with bare coupling $g_{B}=J S^{2} / T$. The prefactor in eq. (5) is the ratio between the renormalization group invariant scales on the lattice and in the $\overline{M S}$ renormalization scheme calculated long time ago in ref. 21.

${ }^{4}$ Unfortunately, some of the most interesting large correlation length MC results at $S=1$ and 5/2 16 are not yet published and missing from this figure.
} 
2D quantum Heisenberg model vs. the classical 2D $O(3)$ non-linear $\sigma$-model: In order to extend the result in eq. (3) we recall that the quantum spin model can be mapped onto the $2 \mathrm{D} \sigma$-model

$$
\mathcal{A}_{\sigma}=\frac{1}{2 g} \int d^{2} x \partial_{i} \mathbf{e}(x) \partial_{i} \mathbf{e}(x), \quad \mathbf{e}^{2}=1, \quad i=1,2
$$

under the condition that the correlation length $\xi$ of the quantum model satisfies $\xi \gg c / T$ and $\xi \gg a$, where $c / T$ is the length scale defined by the temperature. In particular, one is not forced to consider the parameter region only where the leading effective field theory in eq. (2) correctly describes the magnon physics. This is important, since eq. (2) does not give account of the cut-off effects in the quantum Heisenberg model. The cut-off effects are related to the fact that the non-magnon length scales like $c / \rho_{s}$ are not much larger than the lattice unit $a$. Actually, for large $S, c / \rho_{s} \sim 1 / S$ becomes much smaller than $a$. The cut-off effects enter the effective prescription eq. (2) on the 4-derivative level first 9 and contribute to the $O\left(T^{2}\right)$ correction in eq. (3) when $T \rightarrow 0$ with $J$ and $S$ fixed. In this sense eq. (2) and eq. (3) become correct at sufficiently small temperatures for any given $S$. For large $S$, however this happens only at astronomically large correlation lengths, while the mapping to the $2 \mathrm{D} \sigma$-model is valid much earlier.

Let us turn now to the argument concerning the mapping of the $2 \mathrm{D}$ quantum Heisenberg model onto the classical 2D $\sigma$-model. 7 The partition function of the quantum Heisenberg model can be represented in terms of a path integral using coherent states, for example 23. The action is constructed in terms of a 3component classical field $\mathbf{e}\left(\tau, n_{1}, n_{2}\right), \mathbf{e}^{2}=1$, where $n_{1}, n_{2}$ are coordinates of the two-dimensional spatial lattice and $\tau \in(0, c / T)$ is the continuous coordinate of the periodic imaginary time direction. If the correlation length $\xi$ is much larger than $c / T$ (which is the case for small temperatures), in units of $\xi$ we have a thin slab with two infinite space directions. This is just a special regularization of the 2D non-linear $\sigma$-model in eq. (6).

Connecting the parameters of the quantum and the classical models: The mapping discussed above leads to quantitative predictions if we connect the parameters of the quantum Heisenberg model with the coupling $g$ of the non-linear $\sigma$-model. A possibility is to choose a convenient long-distance quantity and calculate it in both models. The predictions should match leading to the connection we are looking for.

A convenient low-energy quantity is the free energy density $f$ as the function of the chemical potential $h .6$ This was the choice in ref. 12, where $f(h)-$ $f(0)$ was calculated in two-loop perturbation theory in the $2 \mathrm{D}$ classical $\sigma$-model

\footnotetext{
${ }^{5}$ For related arguments and different wording, see 12,22 .

${ }^{6}$ Unlike the magnetic field, the chemical potential is renormalization group invariant.
} 
eq. (6) and also in the effective model eq. (2) (after introducing a chemical potential in both models in an equivalent way). Matching the results gives 12

$$
\frac{1}{g(p)}_{\mid p=T / c}=\frac{\rho_{s}}{T}+\alpha+\beta \frac{T}{\rho_{s}}, \quad \alpha=0, \beta=-\frac{3}{4} \frac{1}{(2 \pi)^{2}},
$$

where $g(p)$ is the renormalized coupling constant of the $\sigma$-model at momentum $p$. Using the relation 11]

$$
\xi=\frac{e}{8} \frac{1}{p} \frac{g(p)}{2 \pi} \exp \left(\frac{2 \pi}{g(p)}\right)\left(1+\frac{1}{8 \pi} g(p)+\ldots\right)
$$

eq. (7) leads to the prediction in eq. (3).

Calculating $f(h)-f(0)$ in the quantum Heisenberg model with the help of the effective model in eq. (2) (rather than using the Hamilton operator directly) is an elegant, powerful method. The underlying assumption is that the higher derivative terms in the effective action $\mathcal{A}_{\text {eff }}$, which do not contribute up to the two-loop order 9, give controllable, small corrections. As we discussed before, due to the large cut-off effects at large $S$ in the quantum model, this assumption is true at very low temperatures (astronomically large correlation lengths) only.

In order to calculate the corrections to eq. (7) due to cut-off effects, we have to abandon the effective prescription $[7$ and work with the Hamilton operator directly. We shall use spin-wave expansion (SWE) to derive $f(h)-f(0)$. It is natural to consider $T$ an $O(S)$ quantity when doing thermodynamics in SWE. Then the 3 terms in eq. (7) are $O(S), O(1)$ and $O(1 / S)$. Our SWE runs up to second order and so identifies corrections to the first two terms in eq. (7).

The chemical potential introduced in the classical $\sigma$-model (see, eq. (4) in ref.[12]) corresponds to an imaginary twist in the quantum Heisenberg model]

$$
\begin{aligned}
H(h)= & H(0)+J \sum_{n}\left\{(\cosh (h a)-1)\left(\mathbf{S}_{n+\hat{1}}^{1} \mathbf{S}_{n}^{1}+\mathbf{S}_{n+\hat{1}}^{3} \mathbf{S}_{n}^{3}\right)\right. \\
& \left.-i \sinh (h a)\left(\mathbf{S}_{n+\hat{1}}^{1} \mathbf{S}_{n}^{3}-\mathbf{S}_{n+\hat{1}}^{3} \mathbf{S}_{n}^{1}\right)\right\}
\end{aligned}
$$

Using the Holstein-Primakoff creation and annihilation operators, expanding $H(h)$ for large $S$, keeping the $O\left(S^{2}\right)$ and $O(S)$ terms and performing a Bo-

\footnotetext{
${ }^{7}$ If $T / \Lambda^{\text {cut }}$ is small, the cut-off effects can be taken into account by including 4-derivative terms (with unknown couplings) in eq. (2). For $\rho_{s} \gg T \sim \Lambda^{\text {cut }}$, however, the systematic derivative expansion of chiral perturbation theory breaks down.

${ }^{8}$ The author is indebted to Uwe Wiese for explaining the proper way of introducing the chemical potential into the Heisenberg model.
} 
goljubov transformation 9 we obtained

$$
H(h)=V \epsilon_{0}(h)+\sum_{k} \omega_{k}(h) b_{k}^{\dagger} b_{k}, \quad\left[b_{k}, b_{k^{\prime}}^{\dagger}\right]=\delta_{k, k^{\prime}},
$$

where, in the infinite volume limit

$$
\begin{aligned}
\epsilon_{0}(h) & =-S(S+1) J\left(d+\frac{1}{2}(h a)^{2}\right)+\frac{1}{2} \int \frac{d^{2} k}{(2 \pi)^{2}} \omega_{k}(h), \\
\omega_{k}(h) & =2 J S\left[r_{k}+(h a)^{2} s_{k}\right]^{1 / 2}, \\
r_{k} & =\sum_{i}\left(1-\cos k_{i}\right) \sum_{j}\left(1+\cos k_{j}\right), \\
s_{k} & =\frac{1}{2} \cos k_{1} \sum_{i}\left(1-\cos k_{i}\right)+d, \quad i, j=1,2 .
\end{aligned}
$$

We denoted by $d$ and $V$ the space dimension ( $d=2$ in our case) and volume, respectively. The momentum space integral runs over the Brillouin zone. The simple Hamilton operator in eq. (10) describes free magnon excitations and a zero-point energy. The magnon specie around $k=(0,0)$ picks up a mass $\sim h^{2}$, the one around $k=(\pi, \pi)$ remains massless since $s_{k}$ goes to zero there. It is an easy exercise to show that the contribution of the zero point energy to the free energy per unit slab area $f(h)-f(0)$ is $-h^{2} \rho_{s}^{S W} /(2 T)$, where $\rho_{s}^{S W}$ is the SWE result for the spin-stiffness up to and including $O(S)$. Actually, this should be so in any order of the SWE, due to the fact that $\rho_{s}$ is identical to the helicity modulus and $h$ is an imaginary twist 25, 9. Adding the contribution from the magnon excitations we get:

$$
f(h)-f(0)=-\frac{1}{2} h^{2} \frac{\rho_{s}^{S W}}{T}+\frac{1}{a^{2}} \int \frac{d^{2} k}{(2 \pi)^{2}} \ln \frac{1-\exp \left(-\frac{1}{T} \omega_{k}(h)\right)}{1-\exp \left(-\frac{1}{T} \omega_{k}(0)\right)} .
$$

We have to locate the $O\left(h^{2}\right)$ part of the integral in eq. (12). The result can be written in the following way:

$$
f(h)-f(0)=-\frac{h^{2}}{2}\left\{\frac{\rho_{s}^{S W}}{T}+\frac{1}{2 \pi}\left[\ln \frac{h c^{\mathrm{SW}}}{T}-\frac{1}{2}-C(\gamma)\right]\right\},
$$

where

$$
\begin{aligned}
C(\gamma)= & \frac{\pi}{2}+\ln 8+\ln \gamma \\
& +2 \pi \gamma^{2} \int \frac{d^{2} k}{(2 \pi)^{2}} \frac{s_{k}}{\eta_{k}} \frac{\eta_{k} \exp \left(-\eta_{k}\right)-1+\exp \left(-\eta_{k}\right)}{\eta_{k}\left[1-\exp \left(-\eta_{k}\right)\right]} \mid \eta_{k}=\gamma\left(r_{k}\right)^{1 / 2}
\end{aligned}
$$

\footnotetext{
${ }^{9}$ These are standard manipulations in the literature on SWE[24.

${ }^{10}$ Here and in the following we suppress powers higher than $h^{2}$, since those terms in $f(h)$ are are not universal pieces in the $2 \mathrm{D} \sigma$-model.
} 
using the notation $\gamma=2 J S / T$. For $T \rightarrow 0$, $\exp (-C(\gamma))$ behaves like $1-$ $1.05(1) \gamma^{-2}$ and eq. (13) becomes identical with the spin-wave expanded form of eq. (7) in ref. 12] obtained from the effective theory. (There are additional $\propto\left(T / \rho_{s}\right)^{2}$ corrections to the $O\left(T^{2}\right)$ term in eq. (3), of coarse.) This is an explicit confirmation of the effective action technique.

The new term $\propto C(\gamma)$ in eq. (13) modifies the relation between the parameters giving $\alpha=-C(\gamma) /(2 \pi)$ in eq. (7). Eq. (8) leads then to the result quoted in eq. (画).

Let us close this paper with some remarks. It would be interesting to calculate the next order in the SWE which would test the full 2-loop result for consistency and add subleading cut-off corrections. This calculation seems to be feasible.

In the large $S$ limit the square lattice quantum Heisenberg model becomes identical to the standard lattice regularized $O(3) \sigma$-model for any correlation length. Due to the existence of powerful cluster MC techniques precise correlation length data are available from $\xi=O(1)$ up to $O(200)$ (with finite size scaling techniques even beyond) [26] which can be compared with results in the quantum Heisenberg model from series expansion and MC for large $S$.

This paper is about cut-off effects in the quantum Heisenberg model. We should emphasize, this has nothing to do with the cut-off effects in the 2D non-linear $\sigma$-model. We always considered large correlation lengths and so the cut-off effects in the $\sigma$-model, which are suppressed as $\sim \xi^{-2}$, are negligible.

Acknowledgments The author is indebted to Uwe Wiese for explaining how the chemical potential enters the quantum Heisenberg model in our context. $\mathrm{He}$ is also indebted to Peter Keller-Marxer for making his $S=1$ and $5 / 2 \mathrm{MC}$ data available before publication and also for his help in compiling data from the literature. The author thanks him and Ferenc Niedermayer for the critical reading of the manuscript and Rebecca Christianson, Norbert Elstner, Rajiv Singh and Ruggero Vaia for providing data in numerical form for the figure. While working on this subject, the author enjoyed the warm hospitality of Shanta deAlwis, Tom DeGrand and Anna Hasenfratz at the Dept. of Physics, Boulder. This work was partially supported by the US Department of Energy and by Schweizerischer Nationalfond.

\section{References}


[1] B. Keimer et al., Phys.Rev.B46, 14034 (1992); M. Greven et al., Phys.Rev.Lett.72, 1096 (1994) and Z.Phys.B 96, 465 (1995).

[2] K. Nakajima et al., Z.Phys.B96, 479 (1995); R. J. Birgeneau et al., Phys.Rev.B41, 2514 (1990) and references therein.

[3] R. A. Cowley et al., Phys.Rev.B15, 4292 (1977).

[4] Y. S. Lee et al., Eur.Phys.J B5, 15 (1998).

[5] R. L. Leheny et al., cond-mat/9809178, to appear in Phys.Rev.Lett.82 (1999).

[6] S. Weinberg, Phys.Rev.Lett.17, 616 (1966); J. Gasser and H. Leutwyler, Ann.Phys.(NY)158, 142 (1984) and Nucl.Phys.B250, 465 (1985).

[7] M. E. Fisher and V. Privman, Phys.Rev.B32, 447 (1985); E. Brezin and J. Zinn-Justin, Nucl.Phys.B257 [FS14], 867 (1985).

[8] H. Neuberger and T. Ziman, Phys.Rev.B39, 2608 (1989).

[9] P. Hasenfratz and H. Leutwyler, Nucl.Phys.B343, 241 (1990); P. Hasenfratz and F. Niedermayer, Z.Phys.B92, 91 (1993).

[10] S. Chakravarty, B. I. Halperin and D. R. Nelson, Phys.Rev.B39, 2344 (1989).

[11] P. Hasenfratz, M. Maggiore and F. Niedermayer, Phys.Lett.B245, 522 (1990); P. Hasenfratz, and F. Niedermayer, Phys.Lett.B245, 529 (1990).

[12] P. Hasenfratz, and F. Niedermayer, Phys.Lett.B268, 231 (1991).

[13] B. B.Beard, R. J. Birgeneau, M. Greven and U. -J. Wiese, Phys.Rev.Lett.80, 1742 (1998).

[14] M. Greven, U. -J. Wiese and R. J. Birgeneau, Phys.Rev.Lett.77, 1865 (1996); J.-K. Kim D. P. Landau and M. Troyer, Phys.Rev.Lett.79, 1583 (1997); J.-K. Kim and M. Troyer, Phys.Rev.Lett.80, 2705 (1998).

[15] K. Harada, M. Troyer and N. Kawashima, J. Phys.Soc.Jap.67, 1130 (1998).

[16] B. B. Beard, V. Chudnovsky, P. Keller-Marxer and U. -J. Wiese, in preparation.

[17] N. Elstner et al., Phys.Rev.Lett.75, 938 (1995).

[18] A. Cuccoli et al. J.Phys.Cond.Mat.7, 7891 (1995); A. Cuccoli, V. Tognetti, R. Varia and P. Verruchi, Phys.Rev.Lett.77, 3439 (1996) and Phys.Rev.B56, 14456 (1997). 
[19] A. V. Chubukov and S. Sachdev, Phys.Rev.Lett.71, 169 (1993); A. V. Chubukov, S. Sachdev and J. Ye, Phys.Rev.B49, 11919 (1994); N. Elstner, R. L. Glenister, R. R. P. Singh and A. Sokol, Phys.Rev.B51, 8984 (1995).

[20] A. Sokol, N. Elstner and R. R. P. Singh, cond-mat/9505148.

[21] G. Parisi, Phys.Lett.B92, 133 (1980).

[22] S. Chandrasekharan and U.-J. Wiese, hep-lat/9609042.

[23] J. R. Klauder and B.-S. Skagerstam: Coherent States, World Scientific, 1985.

[24] T. Oguchi, Phys.Rev. 117, 117 (1960); J. Igarashi, Phys.Rev.B46, 10763 (1992); C. J. Hamer, Z. Weihong and J. Oitmaa, Phys.Rev.B50, 6877 (1994).

[25] R. R. P. Singh and D. A. Huse, Phys.Rev.B40, 7247 (1989).

[26] U. Wolff, Nucl.Phys.B334, 581 (1990); B. Alles, G. Cella, M. Dilaver and Y. Gündüc, hep-lat/9808003 and references therein. 




Figure 1: The corrected theoretical prediction for the correlation length normalized by the asymptotic behavior (including the $O(T)$ corrections) in eq. (3) for spin $1 / 2,1$ and $5 / 2$ (the upper, middle and lower solid lines, respectively). Correlation lengths larger than 10 lattice units are considered only. The MC data are from ref. [14] $(S=1 / 2)$, ref. 15 $(S=1)$, the experimental points at $S=5 / 2$ are taken from ref. [5], while the circles come from series expansion 17 . The dotted lines correspond to a semiclassical model [18] which performs well at small correlation lengths but does not have the correct asymptotic behavior.For $\xi_{A S}$, at $S=1 / 2$ the values $\rho_{s}=0.180$ and $c=1.657$ [13, while at $S=1$ and $5 / 2$ the SWE results 24 were used. Similarly, $\gamma$ was connected to $T / 2 \pi \rho_{s}$ with the help of the SWE results. 